\title{
BRANCHED STRUCTURES AND AFFINE AND PROJECTIVE BUNDLES ON RIEMANN SURFACES
}

BY

\author{
RICHARD MANDELBAUM
}

\begin{abstract}
A classification for analytic branched $G$-structures on a Riemann surface $M$ is provided by means of a map $\phi_{G}$ into the moduli spaces of flat $G$-bundles on $M .(G=\mathrm{GA}(1, \mathrm{C})$ or $\mathrm{PL}(1, \mathrm{C})$.) Conditions are determined under which $\phi_{G}$ is injective and these conditions are related to the total branching order of the $G$-structures. A decomposition of the space of analytic branched $G$-structures into a disjoint union of analytic varieties is exhibited and it is shown that $\phi_{G}$ is is fact holomorphic on each such variety.
\end{abstract}

1. Introduction. Continuing the study of branched affine and projective structures on Riemann surfaces begun in [12], we seek to classify such structures by means of their branching orders and their coordinate cohomology classes. In addition we investigate the space of branched structures with fixed branching order and relate this space to that of the space of flat (unstable) projective and affine bundles.

Briefly the contents of this paper are the following. $\$ 2$ develops the notation and terminology to be used and defines the structures to be studied. In $\$ 3$ we exhibit the existence of maps $j_{\nu}(\nu=1,2)$ from $\mathbb{Q}(M)=$ ispace of branched affine structures on $M\}$ and $\mathcal{P}(M)=\{$ space of branched projective structures on $M$ \} (where $M$ is a fixed Riemann surface of genus $g$ ) to $H^{1}(M, G A(1, C)$ ) and $H^{1}(M, \operatorname{PL}(1, \mathbf{C}))$ respectively, where $\mathrm{GA}(m, \mathbf{C})$ and $\mathrm{PL}(m, \mathbf{C})$ are the general affine and projective groups of rank $m$ with coefficients in $\mathrm{C}$ ). We also show that if $\Phi \in H^{1}\left(M, \mathrm{GA}(1, \mathrm{C})\right.$ ) (resp. $H^{1}(M, \mathrm{PL}(1, \mathrm{C})$ ) arises from a flat vector bundle $T \in H^{1}\left(M, S \Delta(2, \mathrm{C})\right.$ ) (resp. $H^{1}(M, \operatorname{SL}(2, \mathrm{C})$ ) (where $S \Delta(2, \mathrm{C}) \subset \operatorname{SL}(2, \mathrm{C})$ is the subset of upper triangular matrices of $\operatorname{SL}(2, \mathrm{C})$. Then $\Phi$ is the coordinate class of some affine (resp. projective) structure on $M . \$ \$ 4$ and 5 then discuss the converse situation of when the coordinate cohomology class of a branched structure does in fact arise from a flat vector bundle. We prove that this is always the case for

Presented to the Society, September 1, 1971; received by the editors March 24, 1972. AMS (MOS) subject classifications (1970). Primary 30A46, 30A52; Secondary 32G15, $30 \mathrm{~A} 58$.

Key words and phrases. Branched structures, Riemann surface, affine structure, projective structure, affine bundle, projective bundle, divisor, affine variety, analytic variety. 
coordinate cohomology classes of affine structures. For cohomology classes of projective structures we show that a sufficient condition that they arise from flat vector bundles is that they possess a nonlocally constant cross-section with even total branching order. We then use the above facts to show that in the projective case if two projective structures determine the same cohomology class and both structures have even total branching order less than or equal to $2 g-2$ then they must be projectively equivalent. In the case where either branching order is even and greater than or equal to $2 g$ we give sufficient additional conditions to guarantee that the structures are projectively equivalent.

For the affine case we similarly prove that the map $j_{1}: \mathscr{Q}(M) \rightarrow H^{1}(M, G A(1, \mathbf{C}))$ is injective when restricted to structures with total branching order $2 g-2$. For other branching orders we again give sufficient conditions to guarantee that two affine structures determining the same cohomology class are affinely equivalent and relate these conditions to the distribution of Weierstrass points on $M$.

Lastly in $\$ \$ 6$ and 7 we show that if for any nonnegative integer $k_{,}{ }_{p}{ }_{k} \subset \mathcal{P}(M)$ and ${ }_{a} V_{k} \subset \mathfrak{Q}(M)$ denote the subset of structures of total branching order $k$ then both $a_{k} V_{k}$ and ${ }_{p} V_{k}$ are finite unions of analytic subvarieties and $j_{\nu}(\nu=1,2)$ is holomorphic when restricted to such a subvariety. We thus get an injection $\mu$ of $\bigcup_{i=0}^{i=g-1}{ }_{p} V_{2 i}$ into $H^{1}(M, \operatorname{PL}(1, \mathrm{C}))$ and $a_{2 g-2}$ into $H^{1}(M, \mathrm{GA}(1, \mathrm{C}))$ which is 'almost' holomorphic in the first case and in fact holomorphic in the second.

2. Notation and terminology. We recall some pertinent facts and terminology from [12]. Let $\left\{U_{a}, z_{a}\right\}$ be a coordinate cover for a Riemann surface M. Suppose for each $\alpha, W_{\alpha}: U_{\alpha} \rightarrow Y_{\alpha}$ is a meromorphic function on $U_{\alpha}$ to the open subset $Y_{\alpha}$ of the complex projective line $P$. We note that this implies $W_{\alpha}: U_{\alpha} \rightarrow Y_{\alpha}$ is a locally branched covering map, and we let $O_{f}(p)$ denote the branching order of $f$ at $p$. Suppose further that for each nonempty intersection $U_{a} \cap U_{\beta}$ there exists a meromorphic homeomorphism $\phi_{a \beta}: W_{\beta}\left(U_{a} \cap U_{\beta}\right) \rightarrow W_{\alpha}\left(U_{\alpha} \cap U_{\beta}\right)$ such that $W_{\alpha}=\phi_{a \beta} \circ W_{\beta}$ on $U_{a} \cap U_{\beta}$. Then we shall say $\left\{U_{\alpha}, W_{\alpha}, \phi_{a \beta}\right\}$ is an analytic branched cover on $M$. An analytic branched structure on $M$ is then simply an equivalence class of covers (where two branched covers are equivalent if their union is a branched cover). It is easily verifiable that branching orders are preserved by the above equivalence relation and we can speak of the branch points of a structure.

By adding the restriction that all the homeomorphisms $\phi_{\alpha \beta}$ belong to some pseudo-group $G$ of meromorphic transformations of $\mathbf{P}$ we can also speak of analytic branched $G$-structures on $M$. The only such structures arise when $G=\operatorname{PL}(1, C)$ or when $G=G A(1, C)$ and we call such structures projective or affine branched structures on the Riemann surface $M$. We note that if $M$ has such a branched $G$-structure [U], then we can always choose a representative braoched $G$-cover 
$\left\{U_{a}, W_{a}\right\}$ of $M$ such that each $U_{a}$ has at most one branch point and no branch point $p$ is in two or more distinct $U_{a}$ 's. Furthermore if $U_{a}$ has no branch points then $W_{a}$ is in fact a homeomorphism. We call such a cover a restricted cover on $M$ and henceforth tacitly assume all our covers are restricted.

Now given a branched analytic cover $\left\{U_{a}, W_{a}\right\}$ on $M$ we can canonically associate to it the positive divisor $\mathcal{S}\left(\left\{U_{a}, W_{\alpha}\right\}\right)=\Sigma_{p \in M} O_{W_{\alpha}}(p) \cdot p$ (for some $\alpha$ such that $\left.p \in U_{a}\right)$. If $M$ is compact $\Sigma_{p \in M} O_{w_{\alpha}}(p) \cdot p$ is a finite sum and therefore recalling that branching orders are invariants of the structure represented by $\left\{U_{a}, W_{a}\right\}$ we have a map $\supseteqq:\{$ branched structures on $M\} \rightarrow$ positive divisors on $\left.M\right\}$. Given a positive divisor $\mathscr{D}$ on $M$ we shall say a branched analytic structure is of type $\mathcal{D}$ if and only if, for some representative $\left\{U_{a}, W_{\alpha}\right\}$ of that structure, I $\left\{U_{a}, W_{a}\right\}=\mathscr{I}$. We let $B\left(\left\{U_{a}, W_{a}\right\}\right)=\operatorname{deg} \mathscr{D}=\Sigma_{p \in M} O_{W_{a}}(p)$ and call this the branching order of the structure. We shall also let $\left|\left\{U_{a}, W_{\alpha}\right\}\right|=|\mathfrak{D}|=$ $\operatorname{card}\left\{p_{j} \in M \mid O_{W_{a}}\left(p_{j}\right) \neq 0\right\}$ (i.e. $\left|\left\{U_{a}, W_{a}\right\}\right|$ is the number of distinct points of $D$ ).

Now suppose $\left\{U_{a}, W_{a}\right\}$ is a branched affine structure with a branch point of order $r-1$ at $p_{j}$. Thus, locally in $U_{\alpha} \ni p_{j}$, we have that $W_{\alpha}=\left[g_{\alpha}\left(z_{\alpha}\right)\right]^{ \pm_{r}}$ where $g_{a}: z_{\alpha}\left(U_{\alpha}\right) \rightarrow \mathbf{C}$ is a holomorphic function with $g_{\alpha}(0)=0, g_{\alpha}^{\prime}(0) \neq 0$ and $z_{\alpha}\left(p_{j}\right)=0$. However affine transformations preserve poles so that in contradistinction to the projective case $\left[g_{\alpha}\left(z_{\alpha}\right)\right]^{ \pm r}$ is not equivalent to $\left[g_{\alpha}\left(z_{\alpha}\right)\right]^{-r}$. Thus, in addition to specifying the order of a branch point, we must also specify whether it arises from a pole or not. To this end we introduce the notion of the class divisor $\chi$ of an affine structure as follows:

Suppose $\left\{U_{a}, W_{a}\right\}$ represents an affine structure on $M$ with poles of order $-\nu_{j}$ at $s_{j}(j=1, \ldots, \sigma)$ and holomorphic branch points of order $r_{j}-1$ at $p_{j}$, $j=1, \cdots, n$. (We note that $O_{W_{j}}\left(s_{j}\right)=\left|\nu_{j}\right|-1$.) We now define the class divisor

$$
\chi\left\{U_{a}, W_{a}\right\}=\sum_{j=1}^{n}\left(r_{j}-1\right) \cdot p_{j}+\sum_{j=1}^{\sigma}\left(\nu_{j}-1\right) \cdot s_{j} .
$$

We thus have a map

$$
\chi: \text { (Affine structures on } M) \rightarrow\{\text { Divisors on } M\} \text {. }
$$

Given a divisor $\chi$ on $M$ we say an affine structure represented by $\left\{U_{a}, W_{a}\right\}$ is of class $\chi$ iff $\chi\left\{U_{a}, W_{a}\right\}=\chi$. We let $\rho(\chi)=\operatorname{deg} \chi$ and $|\chi|=$ \{number of distinct points in $\chi\}$ and recall, by Proposition 4 of [12], $\rho\left(\chi\left\{U_{a}, W_{\alpha}\right\}\right)=2 g-2$ for any affine cover $\left\{U_{a}, W_{a}\right\}$. We call a coordinate cover $\left\{U_{a}, W_{a}\right\}$ regular iff $W_{\alpha}$ is into $\mathbf{C}$ for all $a$. Since this property is preserved under affine transformation we shall say $\left\{U_{a}, W_{\alpha}\right\}$ represents a regular affine structure iff it is a regular affine cover. We immediately see that the affine cover $\left\{U_{a}, W_{\alpha}\right\}$ is regular iff $\chi\left\{U_{\alpha}, W_{\alpha}\right\}$ is a positive divisor or equivalently iff $\chi\left\{U_{a}, W_{a}\right\}=D\left\{U_{a}, W_{a}\right\}$.

In what follows we fix $M$ as a compact Riemann surface of genus $g \geq 2$. 
3. Branched structures and flat vector bundles. We now turn to study the relationship between branched structures and bundles on a Riemann surface. Following the notation of [5], [6] suppose $\mathcal{U}=\left\{U_{a}, W_{a}, \phi_{a \beta}\right\}$ represents a projective or affine branched structure on the Riemann surface $M$. We immediately note that the transition functions $\left\{\phi_{\alpha \beta}\right\}$ satisfy $\phi_{\alpha \beta}=\phi_{\beta a}^{-1}$ and $\phi_{\alpha \beta} \phi_{\beta \gamma}=\phi_{a \gamma}$ and therefore define a cocycle in $Z^{1}(\mathcal{U}, \mathrm{PL}(1, \mathrm{C}))$ or $Z^{1}(\mathrm{U}, \mathrm{GA}(1, \mathbf{C}))$. Furthermore equivalent representative covers define equivalent cocycles and this equivalence is preserved under refinement. In the obvious fashion we then have

Lemma 1. There exist canonical mappings $j_{1}$, $j_{2}$ such that

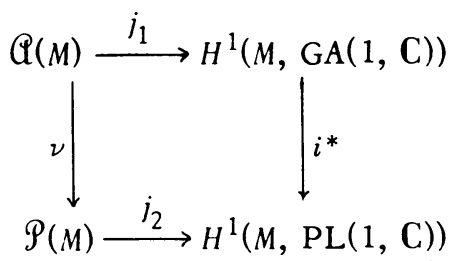

is a commutative diagram (where $\nu: \mathbb{Q}(M) \rightarrow \mathscr{P}(M)$ and $i: \mathrm{GA}(1, \mathrm{C}) \rightarrow \mathrm{PL}(1, \mathbf{C})$ are the natural injections).

We thus see that to each projective (or affine) structure on $M$ there corresponds a flat fiber bundle (or equivalently a coordinate cohomology class). (See [10, p. 41] for details on why this is a bundle.) A flat projective (affine) bundle corresponding in this fashion to some structure on $M$ will then be called an indigenous bundle on $M$. We note that if a flat bundle $\phi$ (projective or affine) on $M$ has a nonlocally flat meromorphic cross-section $\left\{W_{a}\right\}$ then, since $M$ is connected and $W_{\alpha}$ is not constant for some $a,\left\{W_{\alpha}\right\}$ is not constant for all $\alpha$. Thus such a crosssection induces a projective (resp. affine) structure on $M$. We thus see

Lemma 2. Suppose $\phi \in H^{1}(M, \operatorname{PL}(1, \mathrm{C}))\left(H^{1}(M, \mathrm{GA}(1, \mathrm{C}))\right)$. Then $\phi$ is indigenous iff $\phi$ bas a nonlocally flat meromorphic cross-section.

We can proceed further by relating the indigenous projective and affine bundles to flat vector bundles on $M$. We recall the exact sequences

$$
\begin{aligned}
& 0 \rightarrow( \pm I) \rightarrow \operatorname{SL}(2, \mathrm{C}) \stackrel{\mu_{2}}{\longrightarrow} \mathrm{PL}(1, \mathrm{C}) \rightarrow 0 \\
& 0 \rightarrow( \pm I) \rightarrow S \Delta(2, \mathrm{C}) \stackrel{\mu_{1}}{\longrightarrow} \mathrm{GA}(1, \mathrm{C}) \rightarrow 0
\end{aligned}
$$

We now produce the corresponding cohomology diagram

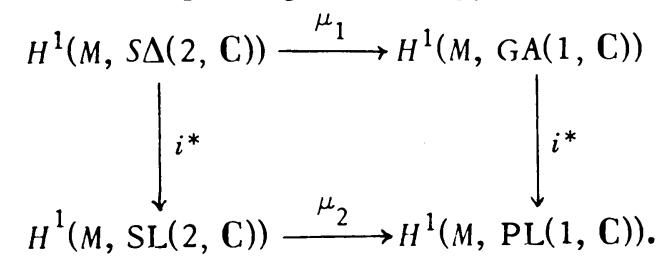


Now if $\phi=\mu_{\nu}(T)(\nu=1,2)$ for some $T \in H^{1}(M, \operatorname{SL}(2, \mathrm{C}))$ (or $H^{1}(M, S \Delta(2, \mathrm{C})$ ) we shall say that $\phi$ is associated to the flat vector (triangular) bundle $T$, or is represented by $T$. We shall then call $\mu_{1}\left(H^{1}(M, S \Delta(2, \mathrm{C}))\right.$ the set of associated flat affine bundle and $\mu_{2}\left(H^{1}(M, \operatorname{SL}(2, \mathrm{C}))\right.$ the set of associated flat projective bundles.

Explicitly (see [6]) if $\phi \in H^{1}\left(M, P L(1, \mathrm{C})\right.$ ) (or $H^{1}(M, \mathrm{GA}(1, \mathrm{C})$ ) we can locally always represent $\phi_{\alpha \beta}$ by $T_{a \beta} \in \mathrm{SL}(2, \mathrm{C})$ ) (or $S \Delta(2, \mathrm{C})$ ) which is uniquely determined up to \pm 1 . The cocycle condition on $\phi$, namely $\phi_{\alpha \beta} \phi_{\beta \gamma}=\phi_{a \gamma}$ when $U_{a} \cap U_{\beta} \cap U_{\gamma} \neq 0$ simply means that $T_{\alpha \beta} T_{\beta \gamma}= \pm T_{\alpha \gamma}$. If we can pick the signs of the various matrices so that the + signs always hold then in fact $\phi$ is an associated bundle.

We then have

Theorem 1. Every associated bundle is indigenous.

Proof. It suffices to show $\mu_{2}^{*}\left(H^{1}(M, \operatorname{SL}(2, \mathrm{C})) \subset j_{2}(P(M))\right.$ since the proof is identical for the affine case. So suppose $\phi \in \mu_{2}^{*}\left(H^{1}(M, \operatorname{SL}(2, \mathrm{C}))\right.$. Thus $\phi$ is an associated bundle on $M$ and for some flat vector bundle $T \in H^{1}(M, \operatorname{SL}(2, \mathrm{C}))$ we have $\phi=\mu_{2}^{*}(T)$. Now by $[5$, Theorems 7 , 9] we have $\mathbb{M}(T) \simeq \mathbb{M} \oplus \mathbb{M}$ where we again recall that $\mathbb{M}(T)$ are the meromorphic cross-sections of $T$, while $\mathbb{N}$ is simply the sheaf of germs of meromorphic functions on $M$. We can thus find two linearly independent meromorphic functions $f_{1}, f_{2}$ such that

$$
\left(F_{a}\right)=\left(\begin{array}{c}
f_{1 a} \\
f_{2 a}
\end{array}\right) \in \Gamma(M, M(T))
$$

is the image of

$$
\left(\begin{array}{l}
f_{1} \\
f_{2}
\end{array}\right) \in \Gamma(M, M \oplus \mathbb{M})
$$

under the induced isomorphism of $\Gamma(M, \mathbb{M}(T)) \simeq \Gamma(M, \mathbb{M} \oplus \mathbb{M})$ and $f_{1 \alpha}, f_{2 a}$ are locally linearly independent (so neither is identically zero).

Now let $W_{\alpha}=f_{1 \alpha} / f_{2 \alpha}$ for each $\alpha$. We note immediately that $W_{\alpha}$ is a nonconstant local meromorphic function on $U_{\alpha}$. Furthermore, if we have

$$
T_{\alpha \beta}=\left(\begin{array}{ll}
a_{\alpha \beta} & b_{\alpha \beta} \\
c_{\alpha \beta} & d_{\alpha \beta}
\end{array}\right),
$$

then in $U_{a} \cap U_{\beta} \neq 0$ we will have

$$
\left(\begin{array}{l}
f_{1 a} \\
f_{2 a}
\end{array}\right)=\left(\begin{array}{l}
a_{\alpha \beta} f_{1 \beta}+b_{\alpha \beta} f_{2 \beta} \\
c_{\alpha \beta} W_{1 \beta}+d_{\alpha \beta} f_{2 \beta}
\end{array}\right) .
$$


Thus

$$
W_{a}=\left(a_{\alpha \beta} W_{\beta}+b_{\alpha \beta}\right) /\left(c_{\alpha \beta} W_{\beta}+d_{\alpha \beta}\right) \text {. }
$$

Thus $\left\{W_{a}\right\}$ represents some $W \in \Gamma(M),((\phi))-\Gamma(M, \mathcal{F}(\phi))$ and so by Lemma $2 \phi$ is indigenous.

For future use if $W \in \Gamma(M, \Re(\phi))$ we shall let $\vartheta_{W}=\Sigma_{p \in M} O_{W}(p) \cdot p$ and $B(W)=\Sigma_{p \in M} O_{W}(p)=B\left(\bigodot_{W}\right)$ be the branching divisor and total branching order of $W$ respectively. We also recall that $O(\phi)$ is the sheaf of holomorphic cross-sections of $\phi$, for any bundle $\phi$.

4. Projective bundles. Having shown that all associated bundles are indigenous we next ask which indigenous bundles are associated. We then have

Theorem 2. Suppose $\phi$ is an indigenous projective bundle with a nonlocally flat holomorphic cross-section

$$
W=\left\{W_{a}\right\} \in \Gamma(M, \Theta(\phi)),
$$

such that $B\left(W_{\alpha}\right)=2 j$ for some integer $j \geq 0$. Then there exists a special linear vector bundle $T \in H^{1}(M, \operatorname{SL}(2, \mathrm{C}))$ such that $\phi$ is associated to $T$. Furthermore there exists a flat line bundle $\mathcal{E}$ and a meromorphic section $k=\left\{\left(k_{1 a}, k_{2 a}\right)\right\} \epsilon$ $\Gamma(M, M(E \otimes T))$ such that $W_{a}=k_{1 a} / k_{2 a}$. If, in addition, $B\left(W_{\alpha}\right) \leq 2 g-2$ then $k$ is a bolomorphic section.

Proof. Since $\phi$ is indigenous we can select a projective cover $\left\{U_{\alpha}, W_{\alpha}, \phi_{\alpha \beta}\right\}$ on $M$ which is mapped into $\phi$. Representing $\phi$ by $\left(\phi_{\alpha \beta}\right) \in Z^{1}(\mathcal{U}, \operatorname{PL}(1, \mathrm{C}))$, for each $\phi_{\alpha \beta}$ we can select a matrix

$$
T_{\alpha \beta}=\left(\begin{array}{ll}
a_{\alpha \beta} & b_{\alpha \beta} \\
c_{\alpha \beta} & d_{\alpha \beta}
\end{array}\right) \in \operatorname{SL}(2, \mathbf{C})
$$

such that

$$
W_{\alpha}(p)=\left(a_{\alpha \beta} W_{\beta}(p)+b_{\alpha \beta}\right) /\left(c_{\alpha \beta} W_{\beta}(p)+d_{\alpha \beta}\right)=\phi_{\alpha \beta} \circ W_{\beta}(p) \text { for } p \in U_{\alpha} \cap U_{\beta} .
$$

We now define $\lambda_{\alpha \beta}(p)=d W_{\beta} / d W_{a}=\left[\phi_{\alpha \beta}^{\prime}\left(W_{\beta}(p)\right]^{-1}\right.$ for $p \in U_{a} \cap U_{\beta}$. We recall that we can write $W_{\alpha}=b_{\alpha}\left(z_{\alpha}\right)$ for some holomorphic $b_{\alpha} \in \mathcal{O}_{z_{\alpha}\left(U_{\alpha}\right)}$ and that

$$
\lambda_{\alpha \beta}(p)=\frac{d W_{\beta} / d Z_{\beta}}{d W_{\alpha} / d Z_{\alpha}}(p)=\frac{b_{\beta}^{\prime}}{b_{\alpha}^{\prime}} \kappa_{\alpha \beta}(p) \quad \text { where } \kappa=\left\{\kappa_{\alpha \beta}\right\}
$$

is the canonical bundle on $M$. Writing $H_{\alpha \beta}=b_{\beta}^{\prime} / b_{a}^{\prime}$ we clearly see that $\left\{H_{\alpha \beta}\right\}$ represents a line bundle $H \in H^{1}\left(M, \Theta^{*}\right)$ and therefore $\lambda_{a \beta}=\kappa_{a \beta} H_{a \beta}$ similarly represents a line bundle $\lambda \in H^{1}\left(M, \Theta^{*}\right)$. Then we have $c\left(\lambda_{\alpha \beta}\right)=c\left(\kappa_{\alpha \beta}\right)+c\left(H_{a \beta}\right)$ $=2 g-2+c\left(H_{a \beta}\right)$ (where $c(\lambda)$ is the Chern class of $\lambda$ ). 
However if we let $f_{\alpha}=\left\{1 / h_{\alpha}^{\prime}\right\}$ we immediately see that $\left\{f_{\alpha}\right\}$ is a cross-section of $\left\{H_{\alpha \beta}\right\}$ and therefore

$$
c\left(H_{a \beta}\right)=\sum_{p \in M} \nu_{p}\left\{f_{\alpha}\right\}=-\sum_{p \in M} \nu_{p}\left\{b_{\alpha}^{\prime}\right\}=-\sum_{p \in M} O_{W_{\alpha}}(p)=-2 j .
$$

Thus $c(\lambda)=2 g-2-2 j=2 m$ where $m \geq 0$ if and only if $B\left(W_{a}\right)=2 j<2 g-2$. Now suppose $m \geq 0$ and select $m$ distinct points $p_{1}, \cdots, p_{m}$ and let $\xi=\Pi_{i=1}^{i=m} \zeta_{p_{i}}^{2}$ where $\zeta_{p_{i}}$ is a point bundle [4, p. 114] and $\xi=1$ if $m=0$. Now write $\xi=\eta \lambda$ for some complex line bundle $\eta$ and since $c(\xi)=2 m=c(\lambda)$ we can assume $\eta \in H^{1}\left(M, \mathbf{C}^{*}\right)$. Since $\xi$ is a product of point bundles $\zeta_{p_{i}}$ we can find a section $g=\left(g_{\alpha}\right) \in \Gamma(M, O(\xi))$ such that $\mathscr{O}(g)=\sum_{i=1}^{i=m} 2 \cdot p_{i}$ (where $g$ is a nonzero constant if $m=0$ ).

Now by computation we see that for $p \in U_{a} \cap U_{\beta}, \lambda_{a \beta}(p)=\left[\phi_{a \beta}^{\prime}\right]^{-1}=$ $\left(c_{\alpha \beta} W_{\beta}(p)+d_{\alpha \beta}\right)^{2}$. Thus the condition that $g$ be a section is just that $g_{a}(p)=$ $\eta_{\alpha \beta}\left(c_{\alpha \beta} W_{\beta}(p)+d_{\alpha \beta}(p)\right)^{2} g_{\beta}(p)$ where $\eta_{\alpha \beta}$ represents $\eta$. Since the divisor of $g$ is even at each point we can select a well-defined branch of $k_{2 \alpha}(p)=\sqrt{g_{\alpha}(p)}$ which is analytic in $U_{a}$ and such that for $p \in U_{a} \cap U_{\beta}$

$$
k_{2 \alpha}(p)=\mathcal{E}_{\alpha \beta} \cdot\left(c_{\alpha \beta} W_{\beta}(p)+d_{\alpha \beta}\right) k_{2 \beta}(p)
$$

where $\mathcal{E}_{\alpha \beta}$ represents a flat line bundle $\mathcal{E}$ such that $\mathcal{E}^{2}=\eta$. Introducing the holomorphic functions $k_{1 \alpha}(p)=W_{\alpha}(p) \cdot k_{2 \alpha}(p)$ we see that

$$
\begin{aligned}
& k_{1 \alpha}(p)=E_{\alpha \beta}\left(a_{a \beta} k_{1 \beta}(p)+b_{a \beta} k_{2 \beta}(p)\right), \\
& k_{2 \alpha}(p)=E_{a \beta}\left(c_{\alpha \beta} k_{1 \beta}(p)+d_{\alpha \beta} k_{2 \beta}(p)\right) .
\end{aligned}
$$

Since $W_{\alpha}$ is not constant, the functions $k_{1 \alpha}, k_{2 \alpha}$ are clearly linearly independent and therefore it follows that the matrices $S_{\alpha \beta}=\mathcal{E}_{\alpha \beta} T_{\alpha \beta}$ satisfy $S_{\alpha \beta} S_{\beta \gamma}=S_{\alpha \gamma}$ and thus represent an element $S \in H^{1}(M, G L(2, \mathrm{C}))$. If we divide $S_{\alpha \beta}$ by the square root of its determinant $=\mathcal{E}_{\alpha \beta}$ we will then have $T_{\alpha \beta}$ representing $T \in H^{1}(M, \operatorname{SL}(2, \mathrm{C}))$ and $\mu_{2}^{*}(T)=\phi$. Furthermore clearly we will have $k=\left(k_{1 \alpha}, k_{2 \alpha}\right)$ $\epsilon \Gamma(M, \mathcal{O}(\mathcal{E} \otimes T))$ with $k_{1 \alpha} / k_{2 a}=W_{a}$.

Now, in the general case of $m$ not necessarily positive, we can still pick a finite number of distinct points $p_{1}, \ldots, p_{k} ; s_{1}, \ldots, s_{l}$ subject to the restriction $l-k=m$. We then let

$$
\xi=\prod_{i=1}^{k} \zeta_{p_{i}}^{-2} \prod_{i=1}^{l} \zeta_{s_{i}}^{2}
$$

Then since $c(\xi)=-2 k+2 l=2(l-k)=2 m$ we will again have $\xi=\eta \lambda$ for some $\eta \in H^{1}\left(M, C^{*}\right)$ and we can find a section $\left\{g_{a}\right\} \in \Gamma(M, \mathbb{M}(\xi))$ such that

$$
\mathscr{J}\left(g_{a}\right)=\sum_{i=1}^{k}-2 \cdot p_{i}+\sum_{j=1}^{l} 2 \cdot s_{j}
$$


The rest of the proof now goes through as before except that $\left\{k_{a}\right\}$ is now a meromorphic rather than a holomorphic section.

We now return to the map $j_{2}: P(M) \rightarrow H^{1}(M, P L(1, \mathrm{C}))$ and consider to what extent a projective bundle determines a specific projective structure.

Theorem 3. Suppose $\left\{U_{\alpha}, w_{\alpha}, \phi_{\alpha \beta}\right\},\left\{U_{\alpha}, \widetilde{w}_{\alpha}, \tilde{\phi}_{\alpha \beta}\right\}$ represent projective structures of type $D_{1}$ and $\mathscr{D}_{2}$ respectively with $B\left(\bigcap_{1}\right)=2 j, B\left(D_{2}\right)=2 k$ for some integers $j, k$, with $2 j \leq 2 g-2$ and $2 k \leq 2 g-2$. Then if $\left\{U_{\alpha}, w_{\alpha}, \phi_{a \beta}\right\}$ and $\left\{U_{\alpha}, \widetilde{w}_{\alpha}, \widetilde{\phi}_{a \beta}\right\}$ are both mapped into the same projective bundle $\phi$ under $j_{2}$, then $\left\{U_{a}, w_{a}\right\}$ is projectively equivalent to $\left\{U_{a}, \widetilde{w}_{a}\right\}$ and $\mathscr{I}_{1}=\mathscr{D}_{2}$.

Proof. Let $\left\{U_{a}, w_{a}, \phi_{a \beta}\right\},\left\{U_{a}, \widetilde{w}_{a}, \tilde{\phi}_{a \beta}\right\}$ be as in the hypothesis of the theorem. Applying Theorem 2 to the indigenous bundle $\phi$ determined by $\left\{U_{\alpha}, w_{a}, \phi_{\alpha \beta}\right\}$ and $\left\{U_{a}, \widetilde{w}_{a}, \tilde{\phi}_{a \beta}\right\}$ we can select a flat vector bundle $T$ represented by $\left(T_{a \beta}\right) \epsilon$ $Z^{1}(\mathcal{U}, \mathrm{SL}(2, \mathrm{C}))$, flat line bundles $\xi, \eta$ represented by $\left(\xi_{\alpha \beta}\right),\left(\eta_{\alpha \beta}\right)$, and holomorphic sections

$$
g=\left\{\left(g_{1 a}, g_{2 a}\right)\right\} \in \Gamma(M, \Theta(\xi \otimes T)), \quad b=\left\{\left(b_{1 a}, b_{2 a}\right)\right\} \in \Gamma(M, \Theta(\eta \otimes T)),
$$

such that

$$
w_{a}(p)=\left(g_{1 a} / g_{2 a}\right)(p) \text { and } \dot{\widetilde{w}}_{a}(p)=\left(b_{1 a} / b_{2 a}\right)(p)
$$

(where we have assumed, without loss of generality, that $\phi_{\alpha \beta}=\tilde{\phi}_{\alpha \beta}$ ).

We now construct the matrices

$$
F_{a}(p)=\left(\begin{array}{ll}
g_{1 a}(p) & b_{1 a}(p) \\
g_{2 a}(p) & b_{2 a}(p)
\end{array}\right) \text { for } p \in U_{a}
$$

which are meromorphic in each $U_{a}$, and we note that

$$
F_{a}(p)=T_{a \beta} \cdot F_{\beta}(p) \cdot\left(\begin{array}{cc}
\xi_{\alpha} & 0 \\
0 & \eta_{a \beta}
\end{array}\right) \text { for } p \in U_{a} \cap U_{\beta} .
$$

Letting $G_{\alpha}(p)=\operatorname{det} F_{\alpha}(p)$ for $p \in U_{\alpha}$ we see immediately that $\left(G_{\alpha}\right) \in \Gamma(M, \pi(\xi \eta))$. But $c(\xi \eta)=0$ so either $G_{a} \equiv 0$ or $\Sigma_{p \in M} \nu_{p}\left(G_{a}\right)=0$. However noting that $w_{a}-\widetilde{w}_{a}$ can be chosen holomorphic for each $\alpha$ we get

$$
\begin{aligned}
\sum_{p \in M} \nu_{p}\left(G_{a}\right) & =\sum_{p \in M} \nu_{p}\left(g_{1 a} b_{2 a}-g_{2 a} b_{1 a}\right)=\sum_{p \in M} \nu_{p}\left(\left(w_{\alpha}-\widetilde{w}_{a}\right) g_{2 a} b_{2 a}\right) \\
& =\sum_{p \in M} \nu_{p}\left(w_{a}-\widetilde{w}_{\alpha}\right)+\sum_{p \in M} \nu_{p}\left(g_{2 a}\right)+\sum_{p \in M} \nu_{p}\left(b_{2 a}\right) \\
& \geq \sum_{p \in M} \nu_{p}\left(g_{2 a}\right)+\sum_{p \in M} \nu_{p}\left(b_{2 a}\right) .
\end{aligned}
$$


But, noting the proof of Theorem 2, we see that

$$
\sum_{p \in M} \nu_{p}\left(g_{2 \alpha}\right)=g-1-j \text { and } \sum_{p \in M} \nu_{p}\left(b_{\alpha}\right)=g-1-k .
$$

Thus $\Sigma_{p \in M} \nu_{p}\left(G_{a}\right) \geq 2 g-2-(j+k)$.

Thus if either $j<g-1$ or $k<g-1$ we have $\Sigma_{p \in M} \nu_{p}\left(G_{\alpha}\right)>0$ so that $G_{\alpha} \equiv 0$.

Therefore $\left(g_{1 \alpha}, g_{2 \alpha}\right)$ and $\left(b_{1 \alpha}, b_{2 \alpha}\right)$ are everywhere linearly dependent and $w_{a}=\widetilde{w}_{a}$ for all $a$.

However if $j=k=g-1$ we note immediately by the proof of Theorem 2 that $g_{2 a}$ and $b_{2 a}$ are holomorphic cross-sections of $\alpha$ and thus constants.

Furthermore $\left\{G_{a}\right\}$ is holomorphic so that either $G_{a} \equiv 0$ or $\xi_{\alpha \beta} \eta_{\alpha \beta} \approx 1$ and $G_{\alpha}$ is also a constant. In either case $G_{a}=\left(w_{\alpha}-\widetilde{w}_{\alpha}\right) g_{2 a} b_{2 a}=$ constant implies $w_{\alpha}-\widetilde{w}_{a}$ is constant for each $\alpha$ and thus $\left\{U_{a}, w_{\alpha}\right\}$ is projectively equivalent to $\left\{U_{a}, \widetilde{w}_{\alpha}\right\}$

We can generalize this result to the case where either $2 j>2 g-2$ or $2 k>2 g-2$ by simply noting that if $w_{a}$ and $\widetilde{w}_{a}$ have a zero of order $m$ at some point $p$, then in fact $\Sigma_{p \in M} \nu_{p}\left(G_{\alpha}\right) \geq 2 g-2-(j+k)+m$. Thus we immediately derive:

Corollary 3.1. Suppose $\left\{U_{a}, w_{\alpha}, \phi_{\alpha \beta}\right\},\left\{U_{\alpha}, \widetilde{w}_{\alpha}, \widetilde{\phi}_{\alpha \beta}\right\}$ represent projective structure of type $\bigcap_{0}+\bigcap_{1}$ and $\bigcap_{0}+\bigcap_{2}$ respectively where $B\left(\bigcap_{0}+\bigcap_{1}\right)=2 j$; $B\left(\mathscr{\vartheta}_{0}+\mathscr{D}_{2}\right)=2 k$ and $B\left(\mathscr{I}_{1}\right) \leq 2 g-2, B\left(\mathscr{I}_{2}\right) \leq 2 g-2$. Suppose there exists $A_{\alpha} \in \mathrm{PL}(1, \mathrm{C})$ such that $A_{\alpha} \circ \phi_{\alpha \beta}=\phi_{\alpha \beta} \circ A_{\beta}$ and $A_{\alpha} \circ w_{\alpha}\left(p_{i}\right)=\widetilde{w}_{\alpha}\left(p_{i}\right)$ for $i=1, \cdots, n$ where $\bigcap_{0}=\sum_{i=1}^{i=n}\left(r_{j}-1\right) \cdot p_{i} \geq 0$. Then $\left\{U_{a}, w_{\alpha}\right\}$ is projectively equivalent to $\left\{U_{\alpha}, \widetilde{w}_{\alpha}\right\}$.

5. Affine bundles. In the case of affine bundles the situation is a bit better than in the projective case. Clearly every affine bundle $\phi$ arising out of an affine cover $\left\{U_{\alpha}, W_{\alpha}, \phi_{\alpha \beta}\right\}$ can be represented by means of a flat triangular bundle $\left\{T_{a \beta}\right\}$ (this follows from a straightforward calculation). Thus every indigenous bundle is associated. We thus turn to the question of to what extent an affine bundle determines a specific affine structure. Letting $\pi_{p}(f)$ be the meromorphic (principal) part of the meromorphic function $f$ in a neighborhood of the point $p$, we have

Theorem 4. Suppose $\left\{U_{a}, w_{a}, \phi_{\alpha \beta}\right\},\left\{U_{a}, \widetilde{w}_{\alpha}, \widetilde{\phi}_{\alpha \beta}\right\}$ represent affine structures of class

$$
\chi=\sum_{j=1}^{n}\left(r_{j}-1\right) \cdot p_{j}+\sum_{j=1}^{\sigma}\left(\nu_{j}-1\right) \cdot s_{j}
$$

and

$$
\tilde{\chi}=\sum_{j=1}^{m}\left(t_{j}-1\right) \cdot q_{j}+\sum_{j=1}^{\sigma}\left(\nu_{j}-1\right) \cdot s_{j}
$$

respectively. 
Then if there exist $A_{\alpha} \in \mathrm{GA}(1, \mathrm{C})$ such that (i) $A_{\alpha} \circ \phi_{\alpha \beta} \circ A_{\beta}^{-1}=\phi$ and (ii) $\Re_{s_{j}}\left(\widetilde{w}_{a}\right)=\Re_{s_{j}}\left(A_{a} \circ w_{a}\right)$ for $j=1, \ldots, \sigma$. Then $\left\{U_{a}, w_{a}\right\}$ is affinely equivalent to $\left\{U_{a}, \widetilde{w}_{a}\right\}$

Before proving this theorem we extract the case of $\sigma=0$ as a separate corollary. We recall that a regular affine structure is one without poles.

Corollary 4.1. Suppose $\left\{U_{a}, w_{a}\right\},\left\{U_{a}, \widetilde{w}_{a}\right\}$ represent regular affine structures with $j_{1}\left\{U_{a}, w_{\alpha}\right\}=j_{1}\left\{U_{a}, \widetilde{w}_{a}\right\}$.

Then $\left\{U_{a}, w_{a}\right\} \sim_{\text {afn }}\left\{U_{a}, \widetilde{w}_{a}\right\}$.

Proof of Theorem 4. We note that for $p \in U_{a} \cap U_{\beta}$ we can write $A_{a} \circ w_{\alpha}(p)=$ $a_{\alpha \beta}\left(A_{\beta} \circ w_{\beta}\right)(p)+b_{\alpha \beta}$ and $\widetilde{w}_{a}(p)=a_{\alpha \beta} \widetilde{w}_{\beta}(p)+b_{\alpha \beta}$ for constants $a_{\alpha \beta} \in \mathbf{C}^{*}$, $b_{\alpha \beta} \in \mathbf{C}$ representing the transition function $\tilde{\phi}_{a \beta}$. Letting $V_{a}=A_{\alpha} \circ w_{a}-\widetilde{w}_{a}$ for any $\alpha$, we see that since $\mathbb{M}_{s_{j}}\left(A_{\alpha} \circ w_{\alpha}\right)=\mathbb{M}_{s_{j}}\left(\widetilde{w}_{\alpha}\right)$ that $V_{\alpha} \in \mathcal{O}_{U_{a}}$. Furthermore for $p \in U_{a} \cap U_{\beta}$ we have $V_{a}(p)=a_{\alpha \beta} V_{\beta}(p)$ so that $\left(V_{\alpha}\right) \in \Gamma(M, C(a))$. But then either $V_{\alpha} \equiv 0$ or $a_{\alpha \beta} \sim 1$ and $V_{\alpha}$ is constant. In either case $A_{a} \circ w_{a}=\widetilde{w}_{\alpha}+K$ for some $K \in \mathbf{C}$ so that $\left\{U_{a}, w_{a}\right\} \sim_{\text {afn }}\left\{U_{a}, \widetilde{w}_{a}\right\}$. Using the methods of [9], [16] we can alter the hypothesis to get

Theorem $4^{\prime}$. Suppose $\left\{U_{\alpha}, w_{\alpha}, \phi_{\alpha \beta}\right\},\left\{U_{\alpha}, w_{\alpha}, \phi_{\alpha \beta}\right\}$ represent affine structures of class $\chi$ and $\tilde{\chi}$ respectively. Suppose further $j_{1}\left\{U_{a}, w_{\alpha}\right\}=j_{1}\left\{U_{a}, \widetilde{w}_{\alpha}\right\}$. Then writing $\chi-\tilde{\chi}$ as the difference of two positive divisors $a_{z}-a_{p}$, if either $a_{z}$ or $a_{p}$ is not a special divisor then $\left\{U_{a}, w_{a}\right\} \sim_{a \mathrm{fn}}\left\{U_{a}, \widetilde{w}_{a}\right\}$.

Proof. We recall that a positive divisor $a$ is a special divisor if and only if there exists a nonconstant meromorphic function $f \in M_{M}$ such that $\mathscr{\sim}(f) \geq-a$.

Now we can assume without loss of generality that $\phi_{a \beta}=\widetilde{\phi}_{\alpha \beta}$. We shall represent $\phi_{\alpha \beta}(x)$ by $a_{\alpha \beta} x+b_{\alpha \beta}$ for some $a_{\alpha \beta} \in \mathbf{C}^{*}, b_{\alpha \beta} \in \mathbf{C}$. We then have for $p \in U_{a} \cap U_{\beta} ; w_{\alpha}(p)=a_{\alpha \beta} w_{\alpha}(p)+b_{\alpha \beta}$ and $\widetilde{w}_{\alpha}(p)=a_{\alpha \beta} \widetilde{w}_{\beta}(p)+b_{\alpha \beta}$. Thus $d \widetilde{w}_{\alpha} / d \widetilde{w}_{p}=a_{\alpha \beta}=d w_{\alpha} / d w_{p}$ and therefore if we let $f_{\alpha}=d w_{\alpha} / d \widetilde{w}_{a}$, we see that in $U_{a} \cap U_{\beta} ; f_{\alpha}=d w_{\alpha} / d \widetilde{w}_{a}=d w_{\beta} / d \widetilde{w}_{\beta}=f_{\beta}$. Thus the family $\left\{f_{\alpha}\right\}$ induces a global meromorphic function $f$ such that

$$
\mathscr{S}(f)=\sum_{p \in M} \nu_{p}\left(w_{a}^{\prime}\right) \cdot p-\sum_{p \in M} \nu_{p}\left(\widetilde{w}_{a}^{\prime}\right) \cdot p=\chi-\tilde{\chi}_{0}
$$

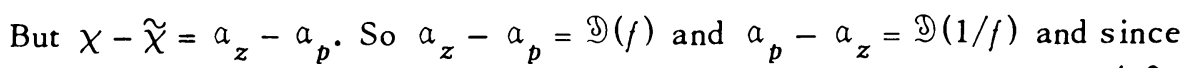
either $a_{z}$ or $a_{p}$ is not a special divisor $f$ must be constant. Thus $d w / d \widetilde{w}_{a}=$ $K_{a} \in \mathrm{C}^{*}$ and so $w_{a}=K_{a} \widetilde{w}_{a}+L_{a}$ for some $L_{a} \in \mathbf{C}$. Therefore $\left\{U_{a}, w_{a}\right\} \sim_{a f n}$ $\left\{U_{a}, \widetilde{w}_{a}\right\}$.

Clearly if $\chi=\tilde{\chi}$ the conclusion of the theorem trivially holds thus giving an alternate proof of Corollary 4.1. Furthermore applying the theorem to the case of divisors of the form $\mathscr{D}=m \cdot p$ we immediately deduce 
Corollary 4.2. Suppose $\left\{U_{a}, w_{\alpha}, \phi_{\alpha \beta}\right\},\left\{U_{a}, \widetilde{w}_{a}, \tilde{\phi}_{\alpha \beta}\right\}$ represent affine structures of class $\chi+m_{1} \cdot p_{1}$ and $\chi+m_{2} \cdot p_{2}$ respectively. Suppose also that $j_{1}\left(\left\{U_{a}, W_{a}\right\}\right)=j_{1}\left(\left\{U_{a}, \widetilde{W}_{a}\right\}\right)$. Then if $r_{i}$ is the first nongap in the Weierstrass gap sequence at $p_{i}$ and if for some $i,\left|m_{i}\right|<r_{1}$ then $\left\{U_{a}, W_{a}\right\} \sim_{a \mathrm{fn}}\left\{U_{a}, \widetilde{W}_{a}\right\}$.

Considering positive divisors of degree $n$ as elements of $S^{n} M$ (the $n$-fold symmetric product of $M$ ) we note that by [13], [14], [15] for $r \leq g$ there exists a proper analytic subset $A \subset S^{r} M$ consisting of all the special divisors of degree $\leq r$. We thus get

Corollary 4.3. Suppose $\left\{U_{\alpha}, w_{\alpha}, \phi_{\alpha \beta}\right\},\left\{U_{\alpha}, \widetilde{w}_{\alpha}, \tilde{\phi}_{\alpha \beta}\right\}$ represent affine structures of class $\chi, \tilde{\chi}$ respectively. Suppose further that $j_{1}\left\{U_{a}, w_{a}\right\}=j_{1}\left\{U_{a}, \widetilde{w}_{a}\right\}$. Then if $\chi-\tilde{X}=a_{z}-a_{p}$ where both $a_{z}$ and $a_{p}$ are positive divisors with $B\left(a_{z}\right)$ $=r_{1} \leq g$ and $B\left(a_{p}\right)=r_{2} \leq g$. Then there exists analytic subsets $A_{1} \subset S^{r_{1}} M$ and $A_{2} \subset S^{r 2} M$ such that if $a_{z} \in S^{r 1} M-A_{1}$ or if $a_{p} \in S^{r 2} M-A_{2}$ then $\left\{U_{a}, w_{a}\right\} \sim_{a f n}$ $\left\{U_{a}, \widetilde{w}_{\alpha}\right\}$.

By a theorem of Meis [13] the set of Riemann surfaces of genus $g(g \geq 2)$ admitting a special divis or of degree less than $[(g+3) / 2]$ (where [ ] is the largest integer function) form a proper analytic subset of the Teichmüller space $M_{g}$ of Riemann surfaces of genus $g$. Thus 'in general' a Riemann surface $M$ of genus $g$ will not have any such special divisors and if either $r_{1}$ or $r_{2}$ in Corollary 4.3 is less than $[(g+3) / 2]$ the conclusion of that corollary will automatically hold on $M$.

We should note that if $r$ is equal to the smallest number of sheets for which $M$ admits a realization over $P$ then $M$ will always have an indigenous bundle $\phi$ such that if $\left\{w_{a}\right\} \in \Gamma(M, \mathcal{M}(\phi))-\Gamma(M, \mathcal{F}(\phi))$ then $B\left\{w_{\alpha}\right\} \geq 2 g-2+2 r$.

For example, the identity bundle of $M$ will not admit nonlocally constant cross sections (i.e. functions) with $B(f)<2 g-2+2 r$. Again therefore by [13] we have that 'in general' (i.e. except for Riemann surfaces contained in a proper analytic subset $A_{g}$ of $M_{g}$ ) a Riemann surface $M$ will always admit an affine bundle $\phi$ such that $\phi$ has no nonlocally constant cross sections $\left\{w_{a}\right\}$ with $B\left(w_{a}\right)<2 g-2$ $+2[(g+3) / 2]$. Thus if $\left\{w_{\alpha}\right\} \in \Gamma(M, \pi(\phi))-\Gamma(M, \mathcal{F}(\phi))$ then $B\left(w_{\alpha}\right) \geq 2 g-2+$ $2[(g+3) / 2] \geq 3 g$. Summing all this up we have

Corollary 4.4. Let $M_{g}$ be the Teichmüller space of Riemann surfaces of genus $g \geq 2$.

Then there exists a proper analytic subset $A_{\boldsymbol{g}}$ of $M_{\boldsymbol{g}}$ such that

(i) If $M \in M_{g_{g}}-A_{g}$ then there exists $\phi \in j_{1}(A(M))$ such that $\left\{w_{a}\right\} \epsilon$ $\Gamma(M, \mathbb{M}(\phi))-T\left(M,{ }^{8} \mathcal{F}(\phi)\right){ }^{8}$ implies $B\left(w_{\alpha}\right) \geq 2 g-2+2[(g+3) / 2] \geq 3 g$, while if

(ii) $M \in A_{g}$ then there exists $\phi \in j_{1}(A(M))$ and an integer $r$ where $2 \leq r<$ $[(g+3) / 2]$ such that 


$$
\left\{w_{a}\right\} \in \Gamma(M, \pi(\phi))-\Gamma(M, \mathcal{F}(\phi)) \Rightarrow B\left(w_{a}\right) \geq 2 g-2+2 r \geq 2 g+2
$$

with $r=2$ only for byperelliptic surfaces.

6. Analytic structure on $\mathscr{P}(M)$. We now return to Lemma 1 for a more thorough study of the spaces $\mathscr{Q}(M), \mathscr{P}(M)$ and the maps $j_{1}, j_{2}$. We begin with $\mathscr{P}(M)$.

Suppose

$$
\mathscr{D}=\sum_{i=1}^{i=B} 1 \cdot S_{i}=\sum_{j=1}^{j=n}\left(r_{j}-1\right) \cdot p_{j}
$$

is a positive divisor on $M$ (where the $p_{j}$ 's are the distinct points among the $S_{i}$ 's and the $r_{j}$ 's are $\geq 2$ ). We then let

$$
\begin{aligned}
& \xi_{\mathbb{D}}=\prod_{i=1}^{i=B} \zeta_{s_{i}}=\zeta_{p_{1}}^{r_{1}-1} \zeta_{p_{2}}^{r_{2}-1} \cdots \zeta_{p_{n}}^{r}{ }^{-1} \\
& \phi_{D}=\prod_{\left\{\text {distinct } s_{i}\right\}} \zeta_{s_{i}}=\zeta_{p_{1}} \zeta_{p_{2}} \cdots \zeta_{p_{n}} .
\end{aligned}
$$

We now form the vector spaces $\Gamma\left(M, \mathcal{O}\left(\kappa^{2} \xi_{\mathfrak{D}}\right)\right)$ and $\Gamma\left(M, \mathcal{O}\left(\kappa^{2} \phi_{\mathfrak{D}}\right)\right)$ and we consider $\Gamma\left(M, \mathcal{O}\left(\kappa^{2} \phi_{\mathcal{D}}\right)\right)$ a subset of $\Gamma\left(M, \Theta\left(\kappa^{2} \xi_{\mathfrak{D}}\right)\right)$ under the natural inclusion induced by the isomorphisms

$$
\begin{array}{r}
\Gamma\left(M, \mathcal{O}\left(\kappa^{2} \xi_{\mathcal{D}}\right)\right) \approx\{\text { meromorphic quadratic differentials } f \\
\text { such that } \operatorname{div}(f)+\mathcal{D} \geq 0\}, \\
\Gamma\left(M, \mathcal{O}\left(\kappa^{2} \phi_{\mathcal{D}}\right)\right) \approx\left\{\begin{array}{r}
\text { meromorphic quadratic differentials } f \\
\text { such that } \left.\operatorname{div}(f)+\sum_{i=1}^{i=n} 1 \cdot p_{i} \geq 0\right\}
\end{array}\right.
\end{array}
$$

where $\operatorname{div}(f)$ is the divisor of $f$. See [4, p. 58] for the isomorphism in question.

Thinking of the positive divisors $\mathscr{D}$ of degree $B$ as points in $S^{B} M$ we can now form the spaces

$$
\begin{aligned}
& B_{B}=\left\{(\mathfrak{I}, x) \mid \mathfrak{D} \in S^{B} M \quad \text { and } \quad x \in \Gamma\left(M, O\left(\kappa^{2} \xi_{\mathfrak{D}}\right)\right)\right\}, \\
& \mathscr{F}_{B}=\left\{(\mathfrak{I}, x) \mid \mathfrak{D} \in S^{B} M \quad \text { and } \quad x \in \Gamma\left(M, O\left(\kappa^{2} \phi_{\mathfrak{D}}\right)\right)\right\}
\end{aligned}
$$

where we consider $\mathscr{F}_{B}$ as a subset of $B_{B}$.

We can also associate to any divisor $\mathscr{D}$ the space ${ }_{p} V_{D}=\{$ projective structures of type $\supseteqq$ on $M\}$ and to any nonnegative integer $B$ the space ${ }_{p} V_{B}=\{(\vartheta, x) \mid \supseteq \in$ $S^{B} M$ and $\left.x \in{ }_{p} V_{\mathcal{D}}\right\} \subset \mathcal{P}(M)$. Under the isomorphism of Theorem 3 of [12] we consider ${ }_{p} V_{D}$ as an affine subvariety of $\Gamma\left(M, \mathcal{O}\left(\kappa^{2} \phi_{\mathcal{D}}\right)\right.$ ) (where we note that by [12, Theorem 3] if $\operatorname{deg} \mathscr{D}=B(\mathscr{D}) \leq 2 g-2$ then ${ }_{p} V_{D}$ contains a $3 g-3$ dimensional submanifold $\left.{ }_{p} V_{\mathcal{D}}^{0}\right)$. Now for any positive integer $B$ let 


$$
\begin{aligned}
& \mathbb{Q}_{B}(M)=\left\{\left(p_{1}, \cdots, p_{B}\right) \in S^{B} M \mid \text { for some } i, j\right. \text { with } \\
& \left.\qquad 1 \leq i, j \leq B \text { and } i \neq j, p_{i}=p_{j}\right\}, \\
& T^{B} M=S^{B} M-\mathbb{Q}_{B} .
\end{aligned}
$$

We then have

Lemma 3. Let $B$ be a positive integer. Then

(1) ${ }_{p} V_{B} \subset \mathcal{F}_{B} \subset B_{B}$ and ${ }_{p} V_{0}=\mathscr{F}_{0}=\mathscr{B}_{0}=\Gamma\left(M, \mathcal{O}\left(\kappa^{2}\right)\right)$.

(2) ${ }_{B} B_{B} \rightarrow S^{B} M$ is a bolomorphic vector bundle with fiber $\mathrm{C}^{3 \mathrm{~g}-3+B}$ and base space $S^{B} M$ under the natural projection map $(\supseteq, x) \rightarrow \supseteq$.

(3) $\mathcal{L}_{B}\left|T^{B} M=\mathscr{B}_{B}\right| T^{B} M$ and therefore $\mathscr{F}_{B} \mid T^{B} M \rightarrow T^{B} M$ is also a bolomorphic vector bundle under the restricted projection map of $B_{B} \rightarrow S^{B} M$.

Proof. Assertion (1) follows directly from the definitions and Corollary 2-1 of [12].

If assertion (2) is true then so is assertion (3), again by simply checking the relevant definitions. We note that $T^{B}(M)$ is a dense open subset of $S^{B} M$ and is in fact simply $S^{B} M-\{$ an affine variety of codim 1$\}$ (i.e. $\mathbb{Q}_{B}(M)$ is simply a union of intersecting hyperplanes in $S^{B} M$ ). To prove our lemma it suffices to prove assertion (2). So let $\nu_{B}=$ (disjoint) $\bigcup_{\mathfrak{D} \in S B_{M}} \xi_{\mathcal{D}}$. Recalling that for any $\mathscr{D} \in S^{B} M, \xi$ is a holomorphic line bundle over $M$ we note that the techniques of the proof of Theorem 6.1 of [14] applied to $\nu_{B}$ show that $\nu_{B} \rightarrow S^{B} M \times M \rightarrow S^{B} M$ is a complex analytic family of holomorphic line bundles over $M$ indexed by the parameter space $S^{B} M$. But then by Theorem 18.1 of [11], if the value of $\operatorname{dim} \Gamma\left(M, \mathcal{O}\left(\kappa^{2} \xi\right)\right)$ is independent of $\mathscr{D} \in S^{B} M$ then

$$
\text { (disjoint) } \bigcup_{\mathfrak{D} \in S^{B_{M}}} \Gamma\left(M, \mathcal{O}\left(\kappa^{2} \xi_{\mathfrak{D}}\right)\right)=\left\{(\mathfrak{I}, x) \mid \mathfrak{I} \in S^{B} M \text { and } x \in \Gamma\left(M, \mathcal{O}\left(\kappa^{2} \xi_{\mathfrak{D}}\right)\right\}=\mathbb{B}_{B}\right.
$$

is a holomorphic vector bundle over $S^{B} M$ under the natural projection $(\supseteq, x) \rightarrow \supseteq$. However by the Riemann-Roch theorem $\operatorname{dim} \Gamma\left(M, \mathcal{O}\left(\kappa^{2} \xi_{D}\right)\right)=3 g-3+B(\supseteqq)$ and $B(\mathcal{D})=B$.

Now again for any positive integer $B$ let $\operatorname{Part}(B)$ be the set of partitions of $B$ and let $\operatorname{Part}(0)=\{0\}$. If $\sigma \in \operatorname{Part}(B)$ we will represent $\sigma$ as a finite sequence of positive integers $\left(\sigma_{1} \cdots \sigma_{n}\right)(n \geq 1)$ such that $\sum_{i=1}^{i=n} \sigma_{i}=B$. (Note. We are distinguishing between two partitions with different orderings.) We let $|\sigma|$ denote the length of the sequence defining $\sigma$. Now suppose we henceforth identify $S^{n} M$ with a fundamental region in $M^{n}$ isomorphic to it under $\operatorname{Perm}(n)$. (The permutation group on $n$ letters acting on $M^{n}$.) Thus every element $P$ in $S^{n} M$ has a unique representation as $\left(p_{1} \cdots p_{n}\right) \in M^{n}$. Similarly of course, the elements of $T^{n} M$ are also uniquely representable elements in $M^{n}$. Then for any $\sigma \in \operatorname{Part}(B)$ we let $\Sigma^{\sigma} M=\left\{\mathcal{D} \in S^{B} M \mid\right.$ for some $\left.\left(p_{1} \cdots p_{n}\right) \in T^{|\sigma|} M ; \mathcal{D}=\sum_{i=1}^{i=|\sigma|} \sigma_{i} \cdot p_{i}\right\}$ and $\Sigma^{0} M=\{0\}=S^{0} M$.

We shall also let $\sigma_{0} \in \mathrm{Part}(B)$ represent the distinguished partition 
$(1,1, \ldots, 1) \in Z^{B}$ and for any positive integer $1 \leq n \leq B, \operatorname{Part}_{n}(B)=\{\sigma \in \operatorname{Part}(B)\}$ $|\sigma|=n\}$. We immediately see

Lemma 4. Let $B, n$ be positive integers with $1 \leq n \leq B$. Then

(1) $S^{B} M=$ (disjoint) $\bigcup_{\sigma \in \operatorname{Part}^{(B)}} \Sigma^{\sigma} M$.

(2) If $\sigma \in \mathrm{Part}_{n}(B)$ then $\Sigma^{\sigma} M$ is canonically isomorphic to $T^{n} M$.

(3) $\operatorname{Part}_{B}(B)=\left\{\sigma_{0}\right\}$ and $\Sigma^{\sigma}{ }^{0} M$ is canonically isomorpbic to $T^{B} M$.

Proof. All the assertions follow immediately from the definitions involved. We note that the isomorphism of (2) is simply

$$
\mathfrak{g}=\sum_{i=1}^{n} \sigma_{i} \cdot p_{i} \in \Sigma^{\sigma} M \rightarrow\left(p_{1} \cdots p_{n}\right) \in T^{n} M
$$

Definition A. Let $B$ be a nonnegative integer. Let $\sigma \in \mathrm{Part}(B)$.

(1) ${ }_{p} V_{B}(\sigma)=\left\{(\vartheta, x) \in{ }_{p} V_{B} \mid \fallingdotseq \in \Sigma^{\sigma} M\right\}={ }_{p} V_{B} \mid \Sigma^{\sigma} M$.

(2) $\mathscr{F}_{B}(\sigma)=\left\{(D, x) \in \mathscr{F}_{B} \mid \supseteqq \in \Sigma^{\sigma} M\right\}=\mathscr{F}_{B} \mid \Sigma^{\sigma} M$.

(3) $B_{B}(\sigma)=\left\{(\supseteqq, x) \in B_{B} \mid \supseteqq \in \Sigma^{\sigma} M\right\}=\mathscr{B}_{B} \mid \Sigma^{\sigma} M$.

An immediate consequence of Lemma 4 and Definition $A$ is

Lemma 5. Let $B$ be a nonnegative integer. Then

(1) ${ }_{p} V_{B}=$ (disjoint) $\bigcup_{\sigma \in \operatorname{Part}(B)}{ }_{p} V_{B}(\sigma)$

(2) $\mathscr{\digamma}_{B}=$ (disjoint) $\bigcup_{\sigma \in \operatorname{Part}(B)} \mathscr{\digamma}_{B}(\sigma)$

(3) $B_{B}=\mathscr{F}_{B}\left(\sigma_{0}\right) \cup$ (disjoint) $\bigcup_{\sigma \in \operatorname{Part}(B)-\left\{\sigma_{0}\right\}} \mathbb{B}_{B}(\sigma)$

We now get

Theorem 5. Let $B, n$ be positive integers with $1 \leq n \leq B$ or $B=n=0$. Suppose $\sigma \in \mathrm{Part}_{n}(B)$ (or $\sigma \in \mathrm{Part}(0)$ if $B=0$ ). Then

(1) $\mathcal{F}_{B}(\sigma)$ is isomorpbic to $\mathcal{F}_{n} \mid T^{n} M$ and thus bas the structure of a complex analytic vector bundle with base space $T^{n} M$ and fiber $\mathrm{C}^{3 \mathrm{~g}-3+n}$. Furthermore $\mathfrak{F}_{B}\left(\sigma_{0}\right)$ is a dense open subset of $B_{B}$.

(2) ${ }_{p} V_{B}(\sigma)$ is a complex analytic subvariety of $\mathcal{F}_{B}(\sigma)$ and ${ }_{p} V_{B}\left(\sigma_{0}\right)$ is dense in ${ }_{p} V_{B}$ while if $\sigma \neq \sigma_{0},{ }_{p} V_{B}(\sigma)$ is nowbere dense in ${ }_{p} V_{B}$.

(3) ${ }_{p} V_{B}^{0}(\sigma)=\left\{\right.$ regular points of $\left.{ }_{p} V_{B}(\sigma)\right\}$ is a complex analytic submanifold of $\mathrm{F}_{B}(\sigma)$ sucb that

$$
\text { (disjoint) } \bigcup_{\mathcal{D} \in \boldsymbol{\Sigma}^{\sigma} M}{ }_{p} V_{\mathcal{D}}^{0} \subset_{p} V_{B}^{0}(\sigma) \text {. }
$$

Furtbermore if $B \leq 3 g-2$ then $\operatorname{dim}{ }_{p} V_{B}^{0}(\sigma)=3 g-3+B$ and if $B \leq 2 g-2$ then $\pi\left({ }_{p} V_{B}^{0}(\sigma)\right)=\Sigma^{\sigma} M$ where $\pi: \mathscr{F}_{B}(\sigma) \rightarrow \Sigma^{\sigma} M$ is the natural projection map.

Proof. We assume $B \neq 0$ as the case of $B=0$ is trivial.

Proof of (1). By the construction of $\mathscr{T}_{B}(\sigma)$ we see that the canonical isomorphism of $\Sigma^{\sigma} M$ and $T^{n} M$ of Lemma $4(2)$ induces in turn a canonical isomorphism 
of $\mathscr{F}_{B}(\sigma)$ and $\mathscr{F}_{n} \mid T^{n} M$. However, by Lemma 3(3), this shows $\mathscr{F}_{B}(\sigma)$ has the desired bundle structure. Furthermore in the proof of Lemma 4 we note that $T^{B} M$ is a dense open subset of $S^{B} M$ and therefore $\mathscr{F}_{B}\left(\sigma_{0}\right)=\mathscr{F}_{B}\left|T^{B} M=\mathscr{B}_{B}\right| T^{B} M$ is in fact also a dense open subset of $B_{B}$.

Proof of (2). We begin with a lemma.

Lemma 6. Suppose $x \in M$. Then there exists a neighborbood $U_{x}$ of $x$ in $M$ and bolomorphic functions $f, g$ on $U$ (into $T\left(M, \pi\left(\kappa^{2}\right)\right.$ ), the space of meromorphic quadratic differentials on $M)$ such that, for $p \in U$,

(1) $f(p) \in \Gamma\left(M, \mathcal{O}\left(\kappa^{2} \zeta_{p}^{2}\right)\right)$ and if we consider $\Gamma\left(M, \mathcal{O}\left(\kappa^{2} \zeta_{p}^{2}\right)\right)$ as the space of quadratic differentials $q$ with $\operatorname{div}(q) \geq-2 \cdot p$ then $f(p)$ bas the expansion $f(p)(z)=$ $1 / z^{2}+a_{-1} / z+$ (bolomorphic terms) in some neighborbood of $p$ with uniformizer $z$ such that $z(p)=0$.

(2) $g(p) \in \Gamma\left(M, \mathcal{O}\left(\kappa^{2} \zeta_{p}\right)\right)$ and under the identification $\Gamma\left(M, \mathcal{O}\left(\kappa^{2} \zeta_{p}\right)\right) \approx$ \{meromorphic quadratic differential $q$ with $\operatorname{div}(q) \geq-1 \cdot p\} g(p)$ bas the expansion $\alpha / z+$ (bolomorphic terms) with $\alpha \neq 0$; in some neighborbood of $p$ with uniformizer $z$ such that $z(p)=0$.

Proof of Lemma 6. By [17, p. 89] there exists a holomorphic bilinear differential $\Lambda(p, q) d z d \xi$ on $M$ (where $z$ is a uniformizer at $p, \xi$ a uniformizer at $q$ ) such that if $p$ is near $q$ then locally as a function of $z$ we can write

$$
\Lambda(p, q)=1 /[z(p)-z(q)]^{2}+\text { holomorphic terms. }
$$

Now for any $x \in M$ let $\omega d z$ be a holomorphic differential on $M$ such that for some neighborhood $U_{x}$ of $x$ in $M, \omega$ does not vanish on $U_{x}$. (Such a differential always exists by $[4, \mathrm{p} .120]$.) Then for any $p \in U_{x}$ let

$$
f(p)=\Lambda(p, q) d z d \xi \frac{\omega(q) d \xi}{\omega(p) d z}=\frac{\Lambda(p, q) \omega(q)}{\omega(p)} d \xi^{2} .
$$

Then we immediately verify that $f(p)$ is a quadratic differential in $\xi(q)$ such that $\operatorname{div}(f(p)) \geq-2 \cdot p$ and $f(p)$ has the expansion $f(p)(\xi)=1 / \xi^{2}(q)+$ holomorphic terms around $\xi(p)=0$. Furthermore, since the genus of $M$ is $>1$, we can find another holomorphic differential $\mu$ on $M$ such that $\omega$ and $\mu$ are linearly independent. Let

$$
\eta(p, q) d z d \xi=\operatorname{det}\left(\begin{array}{ll}
\omega(p) d z & \omega(q) d \xi \\
\mu(p) d z & \mu(q) d \xi
\end{array}\right) \text { for } p, q \in M
$$

Then by a simple calculation $[17$, p. 320] we see that $\eta(p, q)$ is a holomorphic bilinear differential form with a zero of order 1 at $p=q$. Thus for any $p \in U_{x}$ let

$$
g(p)=\Lambda(p, q) \eta(p, q) d \xi^{2}
$$


We then see that as a function of $\xi(q), g(p)$ is a quadratic differential with $\operatorname{div}(g(p))=-1 \cdot p$ and with the expansion $g(p)(\xi)=a / \xi(q)+$ holomorphic terms around $\xi(p)=0$ where $a \neq 0$.

We now finish the proof of (2).

Let $\tau$ be a holomorphic projective connection on M. (For details see [12].) Let $\left(q_{1} \cdots q_{3 g-3}\right)$ be a basis for the holomorphic quadratic differentials on $M$. For any point $p=\left(p_{1} \cdots p_{n}\right) \in T^{n} M$ pick neighborhoods $U_{i}$ of $p_{i}$ in $M$ and functions $f_{p_{i}}, g_{p_{i}}$ on $U_{i}$ satisfying the conclusions of Lemma 6 . Let $\widetilde{U}=\alpha\left(U_{1} \times \cdots \times U_{n}\right)$ (where $\alpha: M^{n} \leftrightarrow S^{n} M$ is the canonical projection). Suppose $p$ is the isomorphism of Lemma $4(2) p: \Sigma^{\sigma} M \leftrightarrow T^{n} M$. Let $U=p^{-1}(\widetilde{U})$. Then for

$$
\mathfrak{I}=\sum_{i=1}^{i=n} \sigma_{i} p_{i}=\sum_{i=1}^{i=n}\left(r_{1}-1\right) \cdot p_{i} \in U
$$

and

$$
(\mathbf{t}, \mathbf{s})=\left(t_{1}, \cdots, t_{n}, s_{1}, \cdots, s_{3 g-3}\right) \in \mathbf{C}^{n+3 g-3}
$$

let

$$
\mu(\mathrm{t}, \mathbf{s}, \mathfrak{D})=\tau+\sum_{i=1}^{n} 1 / 2\left(1-r_{i}^{2}\right) f_{p_{i}}+\sum_{i=1}^{n} t_{i} \cdot g_{p_{i}}+\sum_{i=1}^{3 g-3} s_{i} \cdot q_{i}
$$

Then $\mu(t, s, D)$ is a projective connection of type $\supseteqq$ on $M$ (see [12] for definition and properties of such connections). Furthermore under the isomorphism of $\Gamma\left(M, O\left(\kappa^{2} \phi_{\mathfrak{D}}\right)\right)$ and $\mathrm{C}^{n+3 g-3}$ given by

$$
(\mathrm{t}, \mathrm{s}) \in \mathrm{C}^{n+3 \mathrm{~g}-3} \rightarrow \sum_{i=1}^{n} t_{i} \cdot g_{p_{i}}+\sum_{i=1}^{3 g-3} s_{i} \cdot q_{i}
$$

we can consider $\mu(t, s, \supseteqq)$ as a holomorphic function on $\pi^{-1}(U)=\mathscr{T}_{B}(\sigma) \mid U$. Now by [12, Theorem 3] we have that if $\theta_{2}$ is the Schwarzian differential operator $\theta_{2} f=f^{\prime \prime} / f^{\prime}-3 / 2\left(f^{\prime \prime} / f^{\prime}\right)^{2}$ and $\supseteqq$ is fixed then

${ }_{p} V_{D} \simeq\left\{\right.$ meromorphic solution space of the differential equation $\left.\theta_{2} f=\mu(t, s, D)\right\}$.

Thus

$$
{ }_{p} V_{B}(\sigma) \mid U \simeq\left\{\text { meromorphic solution space of } \theta_{2} f=\mu(\mathrm{t}, \mathrm{s}, \supseteqq) \text { and } \supseteqq \in U\right\} \text {. }
$$

However by the constructions in [12] we have that for a given $\mathscr{D}$ there exists a polynomial $A\left(r_{j}\right) \in \mathbf{C}\left[x_{1} \cdots x_{r_{j}}\right]$ where $(1 \leq j \leq n)$ such that $\theta_{2} f=\mu(\mathrm{t}, \mathbf{s}$, Э) has a meromorphic solution iff

$$
A\left(r_{j}\right)\left(\mu_{-1}^{j}(\mathrm{t}, \mathrm{s}, \supseteqq), \cdots, \mu_{r_{j-2}}^{j}(\mathrm{t}, \mathrm{s}, \mathscr{P})\right)=0 \quad \text { for } j=1, \cdots, n
$$

(where $\mu(t, s, \supseteqq)$ has the Taylor series expansion

$$
\mu(\mathrm{t}, \mathrm{s}, \mathfrak{D})=\frac{\left(1-r_{j}^{2}\right)}{2 z_{j}^{2}}+\frac{\mu_{-1}^{j}(\mathrm{t}, \mathrm{s}, \supseteq)}{z_{j}}+\sum_{i=0}^{\infty} \mu_{i}^{j}(\mathrm{t}, \mathrm{s}, \supseteq) z_{j}^{i}
$$


where $z_{j}$ is a uniformizer at $p_{j}$ and $\left.z_{j}\left(p_{j}\right)=0\right)$. But since $\mu$ depends linearly on $(t, \mathbf{s})$ and holomorphically on $\mathscr{D}$ we see that for any $j$

$$
A\left(r_{j}\right)\left(\mu_{-1}^{j}(\mathrm{t}, \mathrm{s}, \supseteqq), \cdots, \mu_{r_{j-1}}^{j}(\mathrm{t}, \mathrm{s}, \supseteqq)\right)=K_{j}(\mathrm{t}, \mathrm{s}, \supseteqq)
$$

for some function $K_{j}$, holomorphic in $\mathscr{P}$ and polynomial in $(\mathbf{t}, \sigma)$. Thus $K_{j}(\mathrm{t}, \mathbf{s}, \mathfrak{D})$ is a holomorphic function on $\pi^{-1}(U)$ and

$$
{ }_{p} V_{B}(\sigma) \mid U=\left\{x \in \pi^{-1}(U) \mid K_{j}(x)=0 \text { for } j=1, \ldots, n\right\} \text {. }
$$

Thus by $[8, \mathrm{p} .86]_{p} V_{B}(\sigma)$ is a complex analytic subvariety (not necessarily irreducible though) of $\tilde{F}_{B}(\sigma)$. We note that our proof actually shows that if $\Sigma^{[\sigma]} M=$ $\bigcup_{\beta \in \operatorname{Perm}(n)} \Sigma^{\beta(\sigma)_{M}}$ (where $\left.\beta\left(\sigma_{1}, \ldots, \sigma_{n}\right)=\left(\sigma_{\beta(1)}, \cdots, \sigma_{\beta(n)}\right)\right)$ and if ${ }_{p} V_{B}([\sigma])$ $={ }_{p} V_{B} \mid \Sigma^{[\sigma]} M$. Then ${ }_{p} V_{B}([\sigma])$ is in fact itself an analytic variety. This follows from the fact that $K_{j}(\mathrm{t}, \mathrm{s}, \supseteq)$ can be considered as a function of $\supseteqq$ on $U_{1} \times \cdots \times$ $U_{n} \subset M^{n}$ rather than on $U=p^{-1}\left(\alpha\left(U_{1} \times \cdots \times U_{n}\right)\right)$. However $\Sigma^{[\sigma]} M$ is just itself isomorphic to $\left(M^{n} / G\right)-\mathbb{Q}_{n}$ (where $G$ is some subgroup of Perm $(n)$ ) and thus $K_{j}(\mathbf{t}, \mathbf{s}, \mathfrak{D})$ is in fact holomorphic on $\left.\left(U_{1} \times \cdots \times U_{n}\right) / G \supset \Sigma^{[\sigma]} M\right)$.

The other assertions in (2) then follow from Lemma 3 and the fact that $\Sigma^{\sigma_{0}} M$ is dense in $S^{B} M$ while $\Sigma^{\sigma} M$ is nowhere dense in $S^{B} M$ if $\sigma \neq \sigma_{0}$.

Proof of (3). (3) is a direct consequence of Theorem 3 of [12] (including the remark at the end of Theorem 3$)$ and the observation that if $J(K)$ is the Jacobian matrix of the map

$$
(\mathrm{t}, \mathrm{s}, \supseteqq) \stackrel{K}{\longrightarrow}\left(K_{1}(\mathrm{t}, \mathrm{s}, \supseteqq), \ldots, K_{n}(\mathrm{t}, \mathrm{s}, \supseteqq)\right)
$$

while, if for fixed $\mathscr{D}, J\left(K_{\mathfrak{D}}\right)$ is the Jacobian matrix of the map

then

$$
(\mathrm{t}, \mathrm{s}) \stackrel{K_{D}}{\longrightarrow}\left(K_{1}(\mathrm{t}, \mathrm{s}, \supseteqq), \ldots, K_{n}(\mathrm{t}, \mathrm{s}, \supseteqq)\right),
$$

$$
n \geq \operatorname{rank} J(K) \text { at } \mathcal{D} \geq \operatorname{rank} J\left(K_{\mathfrak{D}}\right),
$$

and again by the remarks in the proof of Theorem 3 of [12] 'in general' rank $J\left(K_{\mathfrak{D}}\right)=n$ (i.e. except on a proper analytic subvariety of $\mathrm{C}^{3 g-3+n}$ ).

Since our subvarieties ${ }_{p} V_{B}(\sigma)$ are contained in $\mathcal{P}(M)$ we can examine what $j_{2}$ does to elements of a fixed ${ }_{p} V_{B}(\sigma)$. We begin by giving an alternative characterization of $j_{2}$ which more clearly reveals its properties. So suppose $\mathbb{U}=\left\{U_{a}, w_{\alpha}\right\}$ represents a projective structure in some ${ }_{p} V_{B}(\sigma)$. Lift $U$ to a projective cover $\mathcal{U}=\left\{\pi^{-1}\left(U_{a}\right), w_{a} \pi\right\}$ of $\tilde{M}$, the universal covering surface of $M$, by means of the natural projection $\pi: \widetilde{M} \rightarrow M$. Since $\tilde{M}$ is simply connected we have by [1, p. 215] that the projective structure on $\widetilde{M}$ represented by $\widetilde{U}$ has another representation $\mathcal{O}=\left\{V_{a} \rho_{a}\right\}$ where all the transition functions are identity maps. Thus the $\left\{\rho_{a}\right\}$ 
actually define a global mapping $\rho: \widetilde{M} \rightarrow P$. Furthermore as in $[4$, p. 217] there exists a homomorphism $\rho_{*}: \pi_{1}(M) \rightarrow \operatorname{PL}(1, \mathbf{C})$ related to $\rho$ by $\rho(T x)=\rho_{*}(T) \cdot \rho(x)$ for any $x \in \widetilde{M}$ and $T \in \pi_{1}(M)$ (where we consider $\pi_{1}(M)$ as a group of covering transformations of $\widetilde{M}$ ). Furthermore if $\mathcal{U}^{\prime}=\left\{U_{a}, w_{a}^{\prime}\right\}$ is a projective cover on $M$ projectively equivalent to $\mathcal{U}$, then if $\left(\rho^{\prime}, \rho_{*}^{\prime}\right)$ are the corresponding maps associated to $U^{\prime}$ we have $\rho^{\prime}=R \circ \rho$ and $\rho_{*}^{\prime}=R \circ \rho_{*} \circ R^{-1}$, for some $R \in \mathrm{PL}(1, \mathrm{C})$. Thus to any projective structure [U] in some ${ }_{p} V_{B}(\sigma)$ we can associate a unique element $\left[\rho_{*}(\mathrm{U})\right]$ in $\operatorname{Hom}\left(\pi_{1}(M), \operatorname{PL}(1, \mathrm{C})\right) / \mathrm{PL}(1, \mathrm{C})$ (where $\mathrm{PL}(1, \mathrm{C})$ acts on $\operatorname{Hom}\left(\pi_{1}(M), \operatorname{PL}(1, \mathrm{C})\right)$ by inner automorphism). However under the identification of $H^{1}(M, \operatorname{PL}(1, \mathrm{C}))$ with $\operatorname{Hom}\left(\pi_{1}(M), \operatorname{PL}(1, \mathrm{C})\right) / \operatorname{PL}(1, \mathrm{C})$ of $[4, \mathrm{PP} .186 ; 185]$ $\left[\rho_{1}(\mathcal{U})\right]$ is simply $j_{2}([\mathrm{U}])$. Furthermore the identification of $H^{1}(M, \operatorname{PL}(1, \mathrm{C}))$ with $\operatorname{Hom}\left(\pi_{1}(M), \mathrm{PL}(1, \mathrm{C})\right) / \mathrm{PL}(1, \mathrm{C})$ provides $H^{1}(M, \mathrm{PL}(1, \mathrm{C}))$ with the structure of a quotient space of a complex analytic variety under a complex Lie group of transformations acting on that variety. More explicitly the space $\operatorname{Hom}\left(\pi_{1}(M), \mathrm{PL}(1, \mathrm{C})\right)$ can be identified with the analytic subvariety $R$ of the complex manifold $(\operatorname{PL}(1, \mathrm{C}))^{2} g$ (where $g=$ genus of $M$ ) given by

$$
R=\left\{\left(S_{1}, \ldots, S_{g}, T_{1}, \ldots, T_{g}\right) \in(\operatorname{PL}(1, \mathrm{C}))^{2} g \mid\left[S_{g}, T_{g}\right] \ldots\left[S_{1}, T_{1}\right]= \pm I\right\}
$$

(where $\left.[S, T]=S T S^{-1} T^{-1}\right)$. PL $(1, \mathrm{C})$ then is a complex Lie group transforming $R$ into itself by $G \cdot\left(S_{1}, \ldots, T_{g}\right) \rightarrow\left(G S_{1} G^{-1}, \ldots, G T_{g} G^{-1}\right)$. If instead of looking at $R / \mathrm{PL}(1, \mathrm{C})$ we let $R_{0}$ be the regular points of $R$ and examine $H^{1}(M, \mathrm{PL}(1, \mathrm{C}))_{0}=R_{0} / \mathrm{PL}(1, \mathrm{C})$ we find it is in fact a complex analytic submanifold of $H^{1}(M, \mathrm{PL}(1, \mathrm{C}))$ of complex dimension $6 g-6$. For more details on the structure of $H^{1}(M, P L(1, C))$ and especially for a proof of the last statement see [3], [5, pp. 193-195].

We now have

Theorem 6. $P(M)$ is the disjoint union of the complex analytic varieties ${ }_{p} V_{B}(\sigma)$ for all nonnegative integers $B$ and all $\sigma \in \operatorname{Part}(B)$. For any such ${ }_{p} V_{B}(\sigma)$

$$
\left.j_{2}\right|_{p} V_{B}(\sigma) \rightarrow H^{1}(M, \text { PL }(1, \mathbf{C})) \text { is bolomorphic. }
$$

Furthermore if ${ }_{p} V=\bigcup_{0 \leq j \leq g-2} V_{2 j}$ then $j_{2}$ is injective on ${ }_{p} V \cup_{p} V_{2 g-2}$ and $j_{2}\left({ }_{p} V\right) \subset H^{1}(M, \mathrm{PL}(1, \mathbf{C}))_{0}$.

Proof. The injectivity of $j_{2}$ on ${ }_{p} V \cup_{p} V_{2 g-2}$ is a simple consequence of Theorem 3 while the structure of $\mathcal{P}(M)$ as a disjoint union of the ${ }_{p} V_{B}(\sigma)$ is obvious. To show $\left.j_{2}\right|_{p} V_{B}(\sigma)$ is holomorphic it suffices (by the discussion preceding the theorem) to show that $\left[\rho_{*}(\mathcal{U})\right]$ depends holomorphically on $[\mathrm{U}] \epsilon_{p} V_{B}(\sigma)$. However noting the discussion in [14, Chapter 6] it suffices to show that $[\rho(\mathcal{U})]$ depends holomorphically on [U]. But locally the map $\rho[\mathcal{U}]$ is given by $T_{a_{j}}{ }^{\circ} w_{a} \circ \pi$ for 
some $T_{a_{j}} \in \mathrm{PL}(1, \mathbf{C})$ and some $\alpha$. Thus it suffices to show that the maps $\left\{w_{\alpha}\right\}$ of $\mathcal{U}$ depend holomorphically on the points in some open subset of ${ }_{p} V_{B}(\sigma)$. However the maps $\left\{w_{a}\right\}$ arise precisely as solutions of the equation

$$
\left\{\theta_{2} w_{\alpha}=\mu_{\alpha}(\mathbf{t}, \mathbf{s}, \supseteq)\right\} \quad \text { for }(\mathbf{t}, \mathbf{s}, \supseteqq) \epsilon_{p} V_{B}(\sigma),
$$

where $\left\{\mu_{a}(t, s, D)\right.$ is the projective connection corresponding to the structure [U] under the identification of [12, Theorem 3]. But by the proof of Theorem 5 we see that $\mu_{a}$ depends holomorphically on the points $(t, s, D)$ in some open subset $N_{[u]}$ of ${ }_{p} V_{B}(\sigma)$. Thus by [2, Chapter 7] the $\left\{w_{\alpha}\right\}$ 's also depend holomorphically on the points in $N_{[u]}$ and so $j_{2}$ is holomorphic on ${ }_{p} V_{B}(\sigma)$ as claimed.

To conclude our proof we must show that $j_{2}\left({ }_{p} V\right) \subset H^{1}(M, \operatorname{PL}(1, \mathrm{C}))_{0}$. However, by [3], [5, p. 184], it suffices to show that if $\Phi \epsilon j_{2}\left({ }_{p} V\right)$ then $\Phi$ is irreducible. However if $\phi \in j_{2}\left({ }_{p} V\right), \Phi$ must have a nonlocally flat meromorphic cross-section $w_{\alpha}$ with $B\left(w_{\alpha}\right) \leq 2 g-4$. But then as the following lemma shows $\Phi$ must be irreducible.

Lemma 7. Suppose $\phi$ is an indigenous affine bundle with a nonlocally constant meromorphic cross-section $w_{\alpha}$. Then for some $m \geq 0, B\left(w_{\alpha}\right)=2 g-2+2 m$ (with $m=0$ implying that $w_{\alpha}$ is holomorphic).

In particular if $\psi$ is an indigenous projective bundle with a nonlocally constant meromorphic cross-section $\nu_{a}$ such that $B\left(\nu_{a}\right)<2 g-2$ then $\psi$ is irreducible.

Proof. Let $\phi, w_{a}$ be as in the hypothesis and form the branched affine cover $\left\{U_{a}, w_{a}\right\}$ on $M$. We then have that the class divisor $\chi$ of $\left\{U_{a}, w_{a}\right\}$ can be written in the form

$$
\chi\left\{U_{\alpha}, w_{\alpha}\right\}=\sum_{j=1}^{j=n}\left(r_{j}-1\right) \cdot p_{j}+\sum_{k=1}^{\sigma}\left(\nu_{k}-1\right) \cdot s_{k}
$$

(for distinct points $p_{i}, s_{k}$, and integers $r_{i} \geq 2$ and $\nu_{k} \leq-1$ ). We then have

$$
\begin{aligned}
B\left(w_{\alpha}\right) & =B\left(\left\{U_{\alpha}, w_{\alpha}\right\}\right)=\sum_{j=1}^{j=n}\left(r_{j}-1\right)+\sum_{k=1}^{\sigma}\left(\left|\nu_{k}\right|-1\right) \\
& =\sum_{j=1}^{j=n}\left(r_{j}-1\right)+\sum_{k=1}^{\sigma}\left(\nu_{k}-1\right)-2 \sum_{k=1}^{\sigma} \nu_{k} .
\end{aligned}
$$

But by $[12$, Proposition 4] we have that

$$
\sum_{j=1}^{n}\left(r_{j}-1\right)+\sum_{k=1}^{\sigma}\left(\nu_{k}-1\right)=2 g-2
$$

and in addition $\Sigma_{k=1}^{\sigma} \nu_{k} \leq 0$ so letting $m=-\Sigma_{k=1}^{\sigma} \nu_{k}$ we get $B\left(w_{\alpha}\right)=2 g-2+2 m$ ( $m$ a nonnegative integer). Clearly $m=0$ if and only if $\sigma=0$ in which case $w_{a}$ is holomorphic. 
The latter part of the lemma then follows immediately upon noting that the reducibility of $\psi$ implies its equivalence to an affine bundle $\psi^{\prime}$ for which $\nu_{a}$ would still be a cross-section.

7. Analytic structure of regular affine structures. We now turn to the branched affine structures on $M$. We restrict ourselves to the case of regular affine structures [see $\$ 2$ for definition]. We let

$$
{ }_{a} V^{\mathfrak{D}}=\{\text { space of affine structures of class } \mathscr{D} \text { on } M\}
$$

for any $\supseteqq \in S^{2} g-2 M$.

$$
a^{V}{ }_{2 g-2}=\left\{(\mathfrak{D}, x) \mid \mathscr{D} \in S^{2 g-2} M \quad \text { and } \quad x \in a_{a} V^{\mathcal{D}}\right\} \subset A(M) \text {. }
$$

Noting that $H^{1}(M, \mathrm{GA}(1, \mathrm{C}))$ can be given a complex structure in a manner entirely analogous to that of $H^{1}(M, \operatorname{PL}(1, \mathrm{C}))$ we have

Theorem 7. $a^{V_{2 g-2}}$ can be given the analytic structure of $S^{2 g-2} \mathrm{M} \times \mathrm{C}^{g}$ so that $\left.j_{1}\right|_{a} V_{2 g-2} \rightarrow H^{1}(M, G A(1, \mathrm{C}))$ is an injective bolomorpbic map.

Proof. We recall by [12] that if $\chi \in S^{2 g-2} M$ then ${ }_{a}{ }^{\chi}$ can be identified with the affine connections of class $\chi$ (where an affine connection $\tau$ on $M$ is of class $\chi=\sum_{i=1}^{i=n} x_{i} \cdot p_{i}$ iff $\tau$ has a pole of order 1 at $p_{i}$ with $\operatorname{Res} p_{i}(\tau)=x_{i}$ for $\left.1 \leq i \leq n\right)$. Furthermore if $b$ is an affine connection of class $\chi$ then $\{b+\omega \mid \omega \in \Gamma(M, \mathcal{O}(\kappa))\}=$ $b+\Gamma\left(M, \mathcal{O}^{1,0}\right)$ is the totality of such connections. Thus if $\phi_{1}, \cdots, \phi_{g}$ are a basis for the holomorphic differentials on $M$, we have

$$
{ }_{a} V^{\boldsymbol{x}} \approx\left\{b+\sum_{i=1}^{i=g} t_{i} \cdot \phi_{i} \mid\left(t_{1}, \ldots, t_{g}\right) \in \mathbf{C}^{8}\right\} .
$$

Since the $\phi_{i}$ are independent of $\chi$ and since if $b$ is an affine connection of class $\chi_{1}$ and $k$ is an affine connection of class $\chi_{2}$ and $b=k$ then $\chi_{1}=\chi_{2}$, it suffices to show there exists a global holomorphic function $b$ on $S^{2} g-2 M$ such that for $\chi \in S^{2 g-2}, b_{\chi}$ is an affine connection of class $\chi$. So suppose $\chi=\left(s_{1}, \cdots\right.$, $\left.s_{2 g-2}\right) \in S^{2 g-2} M$. For any point $r \in M$ let $\omega_{\left(s_{i}, r\right)}$ be an abelian differential of the second kind on $M$ with a pole of order 1 and residue -1 at $s_{i}$, a pole of order 1 and residue +1 at $r$ and holomorphic elsewhere. (If $r=s_{i}$ we assume $\omega_{\left(s_{i}, s_{i}\right)}$ is of the first kind.) By [17] such differentials exist and are holomorphic in their dependence on $r \in M$. Now let $\tau$ be an affine connection of class $\chi$ on $M(r$ exists by $[12$, Proposition 4$])$. For any point $\mathscr{D}=\left(p_{1}, \cdots, p_{2 g-2}\right) \epsilon$ $S^{2} g-2 M$, let

$$
b_{\mathcal{D}}=\tau+\sum_{i=1}^{2 g-2} \omega_{\left(s_{i}, p_{i}\right)}
$$


Then a simple calculation shows that $\mathscr{F}_{D}$ is an affine connection of class $\mathscr{D}$ on $M$ (by [12, Lemma 1]) and by its definition is holomorphic on $S^{2} g^{-2} M$. (We note that the structure of $S^{2} g^{-2} M \times \mathrm{C}^{g}$ induced on $a_{2} V_{2-2}$ is done so in a highly noncanonical fashion.)

The proof that $\left.j_{1}\right|_{a} V_{2 g-2}$ is holomorphic is identical to the similar proof for $\left.j_{2}\right|_{p} V_{B}(\sigma)$ of Theorem 6 while the injectivity is simply a restatement of Corollary 4.1. We note that if $g=2$ then since by [5, p. 196] $\operatorname{dim} H^{1}(M, G A(1, \mathrm{C}))=4 g-4$ $=4$ and $\operatorname{dim}{ }_{a} V_{2 g-2}=2 g-2+g=3 g-2=4$ we have that Int $j_{1}\left({ }_{a} V_{2 g-2}\right)$ is a nonempty open subset of $H^{1}(M, G A(1, \mathrm{C}))$. In a forthcoming article we hope to use the structure of $j_{1}\left({ }_{a} V_{2 g-2}\right)$ to study $H^{1}(M, G A(1, \mathrm{C}))$.

\section{REFERENCES}

1. L. V. Ahlfors, Lectures on quasiconformal mappings, Van Nostrand Math. Studies, no. 10, Van Nostrand, Princeton, N. J., 1966. MR $39 \# 4384$.

2. H. Cartan, Elementary theory of analytic functions, Hermann, Paris; AddisonWesley, Reading, Mass., 1963.

3. R. C. Gunning, Analytic structures on the space of flat vector bundles over a compact Riemann surface, Lectures Notes in Math. No. 185, Springer-Verlag, Berlin and New York, 1971, pp. 47-62.

4. - Lectures on Riemann surfaces, Princeton Math. Notes, Princeton Univ. Press, Princeton, N. J., 1966. MR 34 \#7789.

5. - Lectures on vector bundles over Riemann surfaces, Univ. of Tokyo Press, Tokyo; Princeton Univ. Press, Princeton, N. J., 1967. MR 37 \#5888.

6. - Special coordinate coverings of Riemann surfaces, Math. Ann. 170 (1967), 67-86. MR $34 \$ 7790$.

7. - Some non-abelian problems on compact Riemann surfaces, Complex Analysis (Proc. Conf., Rice Univ., Houston, Tex., 1967), Rice Univ. Studies 54 (1968), no. 4, 3948. MR $39 \# 3516$.

8. R. C. Gunning and H. Rossi, Analytic functions of several complex variables, Prentice-Hall Series in Modern Analysis, Prentice-Hall, Englewood Cliffs, N. J., 1965. MR $31 \# 4927$.

9. N. S. Hawley and M. Schiffer, Half-order differentials on Riemann surfaces, Acta Math. 115 (1966), 199-236. MR 32 \#7739.

10. F. Hirzebruch, Topological methods in algebraic geometry, Die Grundlehren der math. Wissenschaften, Band 131, Springer-Verlag, New York, 1966. MR 34 \#2573.

11. K. Kodaira and D. C. Spencer, On deformations of complex analytic structures. II, Ann. of Math. (2) 67 (1968), 403-465.

12. R. Mandelbaum, Branched structures on Riemann surfaces, Trans. Amer. Math. Soc. 163 (1972), 261-275. MR 44 \#5451.

13. Th. Meis, Die minimale Blätterzahl der Konkretisierung einer kompakten Riemannschen Fläche, Schr. Math. Inst. Univ. Münster No. 16 (1960). MR 26 \#5158.

14. H. Rohrl, On holomorphic families of fiber bundles over the Riemannian sphere, Mem. Coll. Sci. Univ. Kyoto Ser A Math. 33 (1960/61), 435-477. MR 24 \#A1728. 
15. H. Rohrl, Holomorphic fiber bundles over Riemann surfaces, Bull. Amer. Math. Soc. 68 (1962), 125-160. MR 26 \#4373.

16. M. Schiffer and N. S. Hawley, Connections and conformal mapping, Acta Math. 107 (1962), 175-274. MR 32 \#202.

17. M. Schiffer and D. C. Spencer, Functionals of finite Riemann surfaces, Princeton Univ. Press, Princeton, N. J., 1964.

DEPARTMENT OF MATHEMATICS, UNIVERSITY OF MASSACHUSETTS, AMHERST, MASSACHUSETTS 01040

Current address: Department of Pure Mathematics, Weizmann Institute, Rehovot, Israel 